\title{
GUATEMALA: RECAMBIO POLÍTICO Y CONFLICTIVIDAD ENDÉMICA
}

Political change and conflict endemic

\section{RENZO LAUTARO ROSAL}

Universidad Rafael Landívar

\begin{abstract}
RESUMEN
Guatemala experimenta un proceso de cambio político, etapa de transición democrática que no se consolida por la sobrevivencia de factores autoritarios que conviven con reglas democráticas, dando como resultado una aparente estabilidad, donde el control político transita de actores tradicionales (partidos políticos, empresariado) a nuevos actores emergentes. Sus anclajes en las estructuras del poder, desde lo local hasta lo nacional, y la acumulación capitalista que combina recursos ilegales con operaciones al amparo de un estado de derecho endeble y el aumento de la riqueza por la vía de los negocios con el Estado, crea un escenario de permanente confrontación, que no se percibe en la superficie pero sí en ámbitos específicos que vulneran los derechos vitales de las personas, usa el miedo como recurso para la inacción colectiva y controla las instituciones públicas.
\end{abstract}

Palabras clave: Guatemala, Otto Pérez Molina, conflictividad, crisis, coyuntura, agenda del cambio, pactos

\begin{abstract}
Guatemala is undergoing a process of political change. The democratic transition fails to consolidate as authoritarian features still survive and coexist with democratic rules, resulting in an apparent stability where political control is transferred from traditional actors (political parties, business community) to new emerging players. The authoritarian features rooted in the structures of power, local and national, together with a capitalist accumulation that combines illegal resources, a weak rule of law, and increasing wealth generated by doing business with the state, provide a scenario of permanent confrontation. This confrontation is not easily perceived, but it is revealed in specific areas where vital rights of people are violated, fear is used as a resource for collective inaction, and public institutions are controlled.
\end{abstract}

Key words: Guatemala, Otto Pérez Molina, Unrest, Crisis, Actual Situation, Change Agenda, Accords 


\section{TEMAS CLAVE DE LA REALIDAD NACIONAL Y SU IMPACTO POLÍTICO}

\section{Cronología de eventos clave}

El año 2012 dio inicio con un nuevo gobierno, encabezado por el Presidente Otto Pérez Molina, propuesto por el Partido Patriota. Su propuesta electoral, denominada Agenda del Cambio, destaca tres pactos, a manera de direccionadores del nuevo equipo de gobierno: i) Pacto de Seguridad, Justicia y Paz, ii) Pacto Hambre Cero; iii) Pacto Fiscal.

\section{Política social}

La coyuntura social fue determinada por la puesta en marcha del Pacto Hambre Cero y los programas sociales, estos últimos continuidad de las iniciativas desarrolladas por el gobierno anterior. El nuevo gobierno priorizó dos características: 1) institucionalización de los programas, 2) impulso de mecanismos para la transparencia.

Aprovechando la correlación favorable en el Congreso de la República, donde la bancada del nuevo partido oficial era la mayoritaria, pocas semanas después de la toma de posesión el Ejecutivo presentó la propuesta para institucionalizar los programas sociales que la gestión anterior se agrupaban en el Consejo de Cohesión Social. De esa cuenta se creó el Ministerio de Desarrollo Social (Mides), cuyo inicio de operaciones solo fue posible en el segundo semestre. En materia de transparencia, el primer desafío consistió en la depuración de los listados de beneficiarios. A partir de junio, el estrenado ministerio absorbió cuatro de los anteriores programas: Mi Bono Seguro, Bolsa Segura, Mi Comedor Seguro, Beca, y Jóvenes Protagonistas. Los dos primeros programas son complemento importante del Pacto Hambre Cero, en el que participan (con recursos y acciones específicas) nueve ministerios, cuatro secretarías y otras dependencias.

El propósito del Pacto Hambre Cero es reducir en 10\% la prevalencia de desnutrición crónica infantil al finalizar 2015, focalizando las intervenciones en 166 municipios priorizados, de acuerdo con los resultados del Tercer Censo de Talla en escolares de primer grado, del 2008/2009. Hambre Cero pretende articular los esfuerzos, planes y proyectos que se realizan en materia de seguridad alimentaria y nutricional por parte de la institucionalidad pública, con especial énfasis en las capacidades que tienen los gobiernos locales.

2012 cerró con la primera fase de institucionalización de los programas sociales, la creación del MIDES y las primeras acciones para mejorar su transparencia. A nivel sustantivo, los resultados aún muestran poco desarrollo y débiles mecanismos de coordinación con los entes rectores de las políticas sociales.

\section{Crisis y conflictos sociales}

\section{Educación}

Ante la serie de descontentos sociales, la medida más utilizada por el gobierno ha sido intentar mecanismos de diálogo para "cortar" las movilizaciones. Poco se ha hecho en 
dar respuesta a las problemáticas de fondo. Desde las movilizaciones realizadas por el sindicato de maestros, el diálogo quedó abierto y dirigentes del magisterio señalan que a partir de las negociaciones se formaron cuatro mesas de trabajo. El magisterio participó en la elaboración de la propuesta "Ley de Carrera Docente", que viene a complementar la propuesta de Formación Inicial Docente.

Sin embargo, el anuncio de la propuesta de reforma a la carrera docente realizada por el Ministerio de Educación, la que plantea una reforma al pensum de estudios y alargar la carrera de tres a cinco años, no fue bien recibida por los estudiantes. La falta de un debate sobre las debilidades de la propuesta del Mineduc, y el deseo de imponerla apresuradamente, hicieron que el movimiento se iniciara con suspensión de clases y tomas de edificios de las normales. La falta de socialización de la propuesta derivó en enfrentamientos entre estudiantes y autoridades educativas, al punto que la discordia derivó en acciones violentas. El año cerró con acciones legales interpuestas por la Asociación de Colegios Privados ante la CSJ y posteriormente rechazadas por la Corte de Constitucionalidad.

\section{Salud}

Durante su primera semana de ejercicio, el gobierno decretó Estado de Calamidad en el área de salud. El Sindicato Nacional de Trabajadores de Salud de Guatemala (SNTSG) y el gobierno firmaron un acuerdo, por el que los trabajadores recibirían un bono por antigüedad. Ese acuerdo no fue cumplido en los plazos establecidos, los trabajadores anunciaron acciones de hecho; en abril los Ministros de Salud y Trabajo acordaron con los dirigentes sindicales que el bono se pagaría a finales de mayo. Las protestas se dejaron sin efecto.

La situación en el sector estatal de salud es extremadamente compleja. Las carencias en los hospitales y centros asistenciales son permanentes, y el gobierno no hace más que remiendos temporales que no brindan soluciones claras. La situación puede estallar en cualquier momento, y de alguna manera el gobierno ha logrado evitar o posponer esa explosión.

\section{Seguridad y justicia}

La otra iniciativa impulsada decididamente desde el inicio del gobierno es el Pacto por la Seguridad, Justicia y Paz, que intenta responder a la principal bandera electoral del Presidente Pérez Molina.

En 2011 la tasa de homicidios en Guatemala fue de 39 por cada 100 mil habitantes. En 2012 se tuvo una baja del 13\%, disminuyendo a 34 por cada 100 mil habitantes. Sin embargo, los desafíos siguen siendo enormes. Guatemala continúa siendo de los países con mayores niveles de violencia del mundo en tiempos de paz.

El plan del Presidente Pérez Molina propuso la creación de las Fuerzas de Tarea Interinstitucionales, catalogado como "plan emergente de seguridad ciudadana", las que fueron parte de las primeras medidas que llevó a cabo la Presidencia. Las Fuerzas 
Gráfico 1: Tasa anual de homicidios por cada 100 mil habitantes

Tasa anual de homicidios por cada 100 mil habitantes

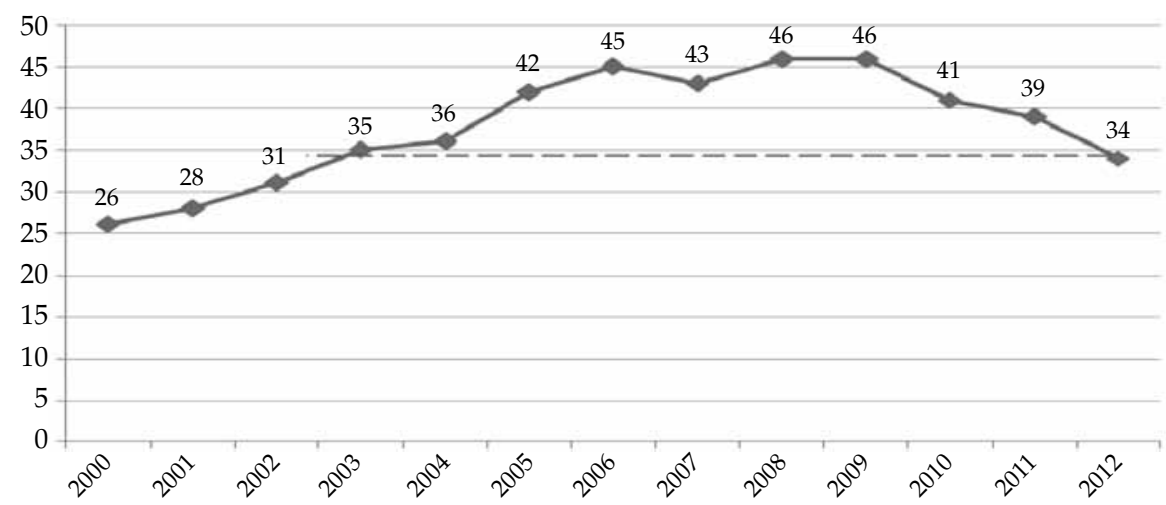

Fuente: Central American Business Intelligence (CABI).

de Tarea fueron lanzadas en enero, dos días después de la toma de posesión, divididas en cinco temas principales: sicariato, secuestros, extorsiones, femicidios, robos y asaltos.

El Pacto por la Seguridad, Justicia y Paz fue presentado en mayo y tiene como fin desarrollar una estrategia cooperativa que incorpore y corresponsabilice a todos los sectores sociales e instituciones del Estado, a fin de legitimar y viabilizar transformaciones y resultados de impacto que permitan alcanzar mejores niveles de gobernabilidad, seguridad y protección frente a la criminalidad, la violencia y la impunidad. En sus avances está la creación del Gabinete Específico por la Seguridad, Justicia y Paz, que tiene a su cargo la propuesta y coordinación de planes, políticas públicas, programas y proyectos para alcanzar mayores niveles de gobernabilidad y seguridad.

En el ámbito normativo e institucional, uno de los avances fue la ratificación del Estado guatemalteco a la Corte Penal Internacional (CPI). El Congreso ratificó el Estatuto de Roma (1998) para adherirse a la Corte Penal Internacional. Se admite que la CPI pueda juzgar delitos de lesa humanidad.

En 2012 la presencia del narcotráfico se intensificó en Centroamérica. Los decomisos, incluso apoyados por operativos del gobierno de Estados Unidos, solo lograron la captura de 85 toneladas, de las 900 que se mueven anualmente en la región. De ellas, solo 3,5 corresponden a Guatemala, cifra muy por debajo de lo logrado en el resto de los países, solo superando a El Salvador.

Aumentó la captura de importantes narcotraficantes locales, lo que permitió que los territorios sean dominados por los carteles mexicanos, principalmente los Zetas, y del Pacífico (alianza entre el de Sinaloa y del Golfo), que expandieron sus operaciones asociados con narcotraficantes locales. Guatemala y Honduras son los dos principales puntos de tráfico. El país ha pasado de ser un lugar de tráfico de drogas, a un centro logístico donde incluso se elaboran drogas sintéticas como anfetaminas. 


\section{Conflictos clave}

\section{Problema}

Conflicto en el Triángulo Ixil, Quiché: amenaza de represión contra campesinos de comunidades del Quiché que mantienen largo conflicto con la familia Brol y la multinacional italiana Enel, que construye la hidroeléctrica Palo Viejo.

Marcha por la Resistencia, la Dignidad, en Defensa de la Tierra y el Territorio: se exige respeto de los territorios y la madre naturaleza en las localidades donde hay empresas mineras, petroleras, hidroeléctricas, construcción de megaproyectos y monocultivos, reclamando también el cese de la persecución contra líderes de comunidades, condonación de los préstamos otorgados por el Fondo de Tierras y aprobación de la Ley de Desarrollo Rural Integral.

Santa Cruz Barillas, Huehuetenango: la comunidad tiene conflictos con la empresa Hidro Santa Cruz S. A., desde 2007, cuando se realizó una consulta popular que rechazó la hidroeléctrica. El asesinato de un líder comunitario causó indignación, que llevó a la comunidad enardecida a tomar el destacamento militar de la zona. Como reacción, el gobierno decretó estado de sitio. Pese a los intentos de retomar el diálogo, en diciembre varios pobladores tomaron el salón municipal como medida de oposición.

San José del Golfo y San Pedro Ayampuc, Guatemala: resistencia pacífica en el lugar conocido como La Puya. Personas que viven en inmediaciones al proyecto minero "Progreso VII Derivada" bloquearon el ingreso de maquinaria para la extracción de oro y plata, para salvaguardar los medios de sobrevivencia de la comunidad. En diciembre se dieron hechos de intimidación hacia la población, donde la Policía Nacional Civil desalojó de manera violenta a pobladores. No se ha logrado entablar una mesa de diálogo.

Instalación de destacamentos militares: en el caso de la base militar en San Juan Sacatepéquez, la medida responde a los conflictos generados entre pobladores y la empresa Cementos Progreso. La base fue inaugurada el 30 de junio, siendo rechazada por una masiva manifestación. El mismo día se inauguró otra en Petén. Otras bases militares fueron o serán instaladas en San Marcos, Quiché, Alta Verapaz e Izabal.

Alaska, Totonicapán: miembros de comunidades indígenas de los 48 cantones de Totonicapán participaron en protestas, produciendo un bloqueo en cinco puntos de las carreteras que comunican con los demás departamentos de la cabecera de Totonicapán. En la Carretera Interamericana alrededor de 70 miembros del ejército llegaron al lugar donde se encontraban los protestantes, quienes empezaron a disparar sin ninguna orden. Son asesinadas siete personas.
Fecha

14 de febrero

19 de marzo

1 de mayo

13 de junio

30 de junio

4 de octubre

Fuente: Elaboración propia.

Según el informe 2012 de la oficina de la ONU contra la Droga y el Delito (UNODC), Centroamérica, sin conflicto bélico, es la región más violenta del mundo, producto del narcotráfico en alianza con las pandillas.

\section{Coyuntura económica}

El año 2012 fue dominado por la lógica de mayores presiones sobre los recursos naturales. Los escenarios de conflictividad a nivel local y las dinámicas sociales de las comunidades 
que más relación tienen con la explotación de los recursos se modificaron continuamente, dando lugar, en muchos casos, a procesos de resistencia pacífica, e incluso a acciones donde el recurso armado, las extorsiones y amenazas, el contubernio de las fuerzas de seguridad y el uso de ejércitos privados fueron herramientas que abonaron en favor de la confrontación permanente.

Centroamérica se debate en un modelo de desarrollo económico basado en la explotación de los recursos naturales bajo regímenes extractivos. Si bien la historia de la región, como la de casi toda Latinoamérica, responde a ese modelo, el presente es más descarnado, evidente y complaciente desde los gobiernos hacia los actores productivos, nacionales y extranjeros. El modelo actual está modificando las dinámicas del capital y del propio modelo empresarial. En la práctica, termina siendo una de las pocas actividades productivas que, además de generar importantes ganancias, contribuye a contrarrestar el notable avance de los empresarios emergentes, especialmente de aquellos cuyas fuentes de riqueza provienen de las redes criminales, entre ellas el narcotráfico.

El modelo de crecimiento acelerado, aunque incipiente en sus dinámicas, marca pautas con dureza por las maneras como se imponen a las resistencias de las colectividades (especialmente indígenas), manipulan a las comunidades, plantean falsas consideraciones acerca de los beneficios que se percibirán, generan un tipo de empleo que contraviene la legislación laboral nacional y los acuerdos internacionales.

La historia de Guatemala solo puede entenderse desde los procesos de acumulación del capital, donde el binomio territorio-comunidades rurales resulta esencial. Esa relación, marcada con nuevos ingredientes, continúa vigente y se fortalece con la nueva vigencia del régimen extractivo, en las que se conjugan dinámicas globales con los procesos de acumulación basados en la modificación de las realidades locales.

Cada uno de los procesos de explotación de los recursos naturales (hidroeléctricas, minería e hidrocarburos) traduce en sus dinámicas las características centrales del modelo extractivo: desterritorialización, reterritorialización, uso y control del territorio, anclaje en el recurso tierra, modificación del mercado laboral, aumento de las vulnerabilidades sociales y afectación de los regímenes de propiedad (privada, comunal). El nuevo gobierno señala, en forma encubierta, la modificación de la concepción y naturaleza del Estado. El modelo de desarrollo que se promueve requiere de un Estado y sus referentes gubernamentales que garanticen los asideros sociales, de economía abierta, de control cultural y políticos para el mantenimiento, sostenibilidad y reacomodo del modelo (capacidad de adaptarse a los cambios y a las tensiones generadas en el ámbito nacional). Esas transformaciones se han acentuado en el primer año de gobierno, cuya fundamentación recae en las nuevas legalidades, las reformas institucionales, la generación de alianzas público-privadas, la focalización de los cuestionamientos como disfuncionalidades, la promoción de los acuerdos comerciales y el control de los procesos locales, como principales determinantes.

Como fórmulas contrarias, nuevas lógicas de resistencia se gestan desde los ámbitos comunitarios, dando lugar a incipientes procesos de surgimiento de nuevos sujetos sociales y políticos. La resistencia, sobrevivencia e incidencia desde lo local, comienza 
a generar conocimientos y estrategias político-comunitarias que emergen con relativa consistencia, pero que aún no alcanzan para promover nuevas formas de representación política.

\section{Reformas fiscales}

Luego de conocer los resultados electorales, en noviembre del 2011, Otto Pérez Molina, por medio de Pavel Centeno, quien operaría como ministro de Finanzas Públicas, impulsó lo que sería la agenda fiscal del nuevo gobierno. Entre varios puntos en esta agenda, destacaba el impulso de una reforma tributaria basada en el pacto fiscal.

Un aspecto que favoreció la negociación entre el Comité Coordinador de Asociaciones Comerciales, Industriales y Financieras (CACIF) y el nuevo gobierno para aprobar la reforma y no fuera bloqueada como ocurrió en el gobierno anterior, fue que el gobierno de Pérez Molina cediera en debilitar la propuesta original del Grupo Promotor del Diálogo Fiscal (GPDF) en algunos puntos. Según el Ejecutivo, esos puntos no distorsionaban excesivamente el contenido de la reforma. Sin embargo, actores especializados en el tema sostienen que las reformas no producirán resultados de incremento de ingresos tributarios en los próximos dos años. Se confirma que el desafío existente respecto de este tipo de reformas obedece a cuestiones puramente políticas. La aprobación de la propuesta a una reforma tributaria que había sido bloqueada durante cuatro años fue aprobada en tiempo récord en 2012. Aprovechando la correlación favorable al Congreso, el 15 de febrero fue aprobada con el voto favorable de 110 diputados (de un total de 158), la Ley de Actualización Tributaria, Decreto 10-2012. La aprobación se llevó a cabo después que las bancadas aliadas al partido oficial, Partido Patriota (PP), consensuaran y modificaran el contenido del proyecto propuesto por el Ejecutivo.

El objetivo de la ley consiste en aumentar la recaudación fiscal para oxigenar las finanzas del actual gobierno, en particular. Se prevé un incremento de la recaudación por aproximadamente cuatro mil quinientos millones de quetzales; cifra considerada inviable para sectores especializados y de la sociedad civil. Las últimas semanas del año se caracterizaron por el inicio de varias reacciones adversas a las reformas, respaldadas por sectores económicos que en principio apoyaron la ley. Se sumaron otras expresiones interponiendo recursos de inconstitucionalidad y otras medidas de presión para que el Ejecutivo diera marcha atrás o al menos reformara los artículos controversiales.

Durante 2012, las autoridades del Ministerio de Finanzas Públicas y de la Superintendencia de Administración Tributaria (SAT) se dieron a la tarea de generar mecanismos para la puesta en marcha de las reformas a partir de enero de 2013. Sin embargo, durante el segundo semestre se generaron fricciones entre los responsables de ambas dependencias, desavenencias que se hicieron públicas. Ese desgaste, donde el Presidente de la República no intervino para su adecuado abordaje interno, devino en la renuncia del titular de la SAT en marzo de 2013.

A pesar del estancamiento de la agenda legislativa, las alianzas partidarias generadas por el partido oficial lograron que el 23 de ese mes se aprobara, de urgencia nacional, el Presupuesto General de Ingresos y Egresos para 2013, por sesenta y seis mil novecientos 
ochenta y cinco mil millones de quetzales (Q. 66.985,4 millones), que supera en 7.430,1 millones el presupuesto actual (59.547,3 millones).

\section{Transparencia}

Según datos de Transparencia Internacional, en Guatemala para el 2010 se da un retroceso en el índice de corrupción de siete posiciones en el ranking mundial, disminuyendo la evaluación en 0,2 puntos. Esta situación empeora en 2011, con el retroceso más grande que ha tenido el país en la historia, con un descenso de 29 posiciones y -0,5 puntos en la evaluación. Estos datos muestran la razón de las acentuadas críticas del Presidente Otto Pérez Molina hacia el gobierno anterior, en cuanto a la poca transparencia de esa gestión.

En marzo del 2012, luego de aprobada la Ley de Actualización Tributaria, se presentó un paquete de 11 leyes de transparencia, el que implica la aprobación de tres nuevas leyes: Ley de Regulación de Fideicomisos Públicos, Ley Contra el Enriquecimiento Ilícito y Ley del Sistema de Planificación y Evaluación de la Gestión Pública. Asimismo, conlleva la reforma de las siguientes leyes: Ley del Organismo Ejecutivo, Ley Orgánica del Presupuesto, Ley del Servicio Civil, Ley de Contrataciones del Estado, Ley de la Contraloría General de Cuentas (CGC) y Ley del Instituto Nacional de Estadística (INE). Sn embargo, hasta el momento el tema sigue siendo impopular en el Congreso y sigue en espera de su aprobación.

La iniciativa de Ley contra el Enriquecimiento Ilícito fue decididamente bloqueada durante más de seis meses por el oficialismo. Sin embargo y favorecida por múltiples expresiones de presión de la sociedad civil, fue aprobada entrando en vigencia el 30 de noviembre. La ley incorpora al Código Penal los delitos de corrupción en oficinas públicas, cobro de comisiones, tráfico de influencias, nombramientos ilegales, cohecho pasivo y activo, además de tipificar las actividades de testaferrato.

Uno de los sucesos que ha golpeado al gobierno de Otto Pérez Molina fue el relacionado con la Empresa Portuaria Quetzal. El gobierno cedió en usufructo terrenos en una operación opaca y cuestionada por diversos sectores sociales y económicos. A pesar de las pruebas que indicaban la opacidad en la negociación, el Ejecutivo defendió el negocio, el que sigue en pie. Expertos jurídicos aseguran que lo actuado es ilegal y que todo apunta a que se trata de una concesión que violó los preceptos de ley.

Durante el 2012, el Fondo Nacional para la Paz (Fonapaz) fue una de las instituciones ampliamente señalada en actos de corrupción. Medios de comunicación informaron sobre la sobrevaloración de compras, las negociaciones y contratos espurios, el reparto de programas con fines clientelares, entre otros desmanes. En forma sorpresiva el Presidente de la República anunció su cierre, pero posteriormente ha cedido a presiones para crear un nuevo fideicomiso que se haga cargo de los programas en curso, generando dudas sobre la contundencia y sentido de la decisión inicial.

En febrero fue creada la Secretaría de Control y Transparencia, la que debía atender instrucciones de la Vicepresidencia de la República para el control y la transparencia, la implementación del gobierno electrónico y garantizar el acceso a la información pública. 
Sin embargo, nueve meses después la Corte de Constitucionalidad suspendió la vigencia del Acuerdo Gubernativo 37-2012, dando lugar a un recurso de inconstitucionalidad presentado por la bancada Libertad Democrática Renovada (LIDER), justificando que esta instancia carece de base legal, y que para su creación era necesario que el Congreso modificara la Ley del Organismo Ejecutivo, y no solo mediante un acuerdo gubernativo.

\section{Coyuntura subregional e internacional}

La propuesta que dominó el escenario internacional fue la sorpresiva propuesta del Presidente de la República para la despenalización de las drogas, lo que reduciría los índices de violencia que afectan a Centroamérica, relacionados en gran parte con el narcotráfico y otros delitos conexos. La propuesta, si bien no abordada en su propuesta electoral, consiste en abrir el diálogo internacional sobre la despenalización de la producción, transporte, comercio y consumo de las drogas, como opción "viable" para derrotar al narcotráfico. El Presidente manifestó, durante el mes de febrero cuando se lanzó la iniciativa, que después de 25 años de las estrategias de lucha contra el narcotráfico es importante abrir un debate para encontrarle una forma mucho más eficaz" a ese flagelo. En principio, la propuesta no encontró eco en los países de la región (con excepción de Costa Rica y Panamá); ese factor solo sirvió para que el gobierno continuara con su labor de difusión y búsqueda de apoyo en otras regiones.

Estados Unidos ratificó tempranamente su oposición a la iniciativa. La secretaria de Seguridad Nacional, Janet Napolitano, manifestó que su país "no considera viable el tema de la despenalización" de las drogas, porque "no es la mejor manera de combatir el narcotráfico". Consideró que "hay mejores maneras de abordar el problema del narcotráfico", como la "reducción de las adicciones", así como en mejorar las capacidades de interceptación de los cargamentos de drogas trasegados por los carteles de narcotraficantes en la región y en "mejorar las capacidades para impedir la producción y distribución de las drogas".

La propuesta fue tomando forma a partir de las presentaciones realizadas en foros de presidentes centroamericanos, diálogos y experiencias de otros países latinoamericanos y espacios de discusión multilaterales. En junio, la propuesta pasó a denominarse "Nuevas Rutas Contra las Drogas para combatir al narcotráfico, una posible despenalización de las drogas en los países centroamericanos". Contiene cuatro ejes principales: a) Endurecimiento en la lucha para la incautación de drogas. El producto que sea incautado deberá ser compensado por los países consumidores; con los recursos generados distribuir el 50 por ciento para combatir al crimen organizado y al narcotráfico, el 25 por ciento en programas para concientizar a la población sobre los daños que genera el consumo de las drogas y otro 25 por ciento en programas de rehabilitación para los adictos; b) Creación de una Corte Penal con jurisdicción regional temporal, que deberá velar únicamente por temas de narcotráfico, tráfico de armas, lavado de dinero y trata de personas, con responsabilidad compartida y diferenciada; c) Despenalización del tránsito de drogas, posición que más ha preocupado a los Estados Unidos, debido al alto consumo de drogas en ese país. La propuesta busca definir y crear un corredor 
de tránsito con propósito único, con controles fronterizos para el debido registro del tránsito de la droga y la creación de una agencia regional para control y el manejo de estupefacientes; d) Buscar otras vías de despenalización. Reportes oficiales indican que el trasiego de cocaína en el corredor regional genera anualmente más de trece mil trescientos veinte millones de dólares.

\section{Coyuntura política}

La suscripción de los Acuerdos de Paz en 1996, anunció la posibilidad de un quiebre en la historia del país y abrió las puertas a la transformación de las instituciones del Estado para que las mismas expresaran y reflejarán las complejas diferencias étnicas, culturales y lingüísticas. Los obstáculos para el cumplimiento de los compromisos mencionados están relacionados con las limitaciones del sistema político guatemalteco, en términos de lograr una efectiva representación de los pueblos indígenas; la heterogeneidad de las organizaciones y corrientes políticas dentro de la población indígena; los problemas que en términos de participación política arrastra la sociedad guatemalteca en su conjunto; y desde una perspectiva de larga duración, el racismo y las lógicas de exclusión.

No obstante, el país ha mejorado en los niveles de participación política, pero todavía existen obstáculos para que esta sea plena y logre integrar a la población que tradicionalmente ha sido excluida. Eso implica asegurar que el sistema y los procesos políticos permitan que la voz de esa población sea escuchada y tengan incidencia real para la elaboración de políticas públicas y la toma de decisiones.

Durante el año 2012 se observaron altos índices de conflictividad, producto del desequilibrio en el relacionamiento entre grupos sociales, el Estado y sus representantes, así como por un sistema político debilitado. Los proyectos de explotación de recursos naturales (hidroeléctricas, minería y las propuestas para incrementar la explotación de hidrocarburos), así como las demandas de organizaciones indígenas y campesinas para lograr la aprobación de la iniciativa de ley 4084, ley del sistema de desarrollo rural integral, fueron los principales detonantes, a los que se sumó el rechazo de diversos sectores a las reformas a la carrera del magisterio (provenientes de estudiantes de las escuelas normales, docentes, padres de familia; a las que se sumaron organizaciones indígenas, campesinas, gremios de colegios privados).

La amplia gama de promesas electorales han comenzado a pasar factura. El primer año de gobierno finalizó con las diversas expresiones de cuestionamiento, ya que siguen sin resolverse las amplias expectativas de la población. Por ejemplo, en materia de explotación de recursos naturales, la gobernabilidad, tanto a escala nacional como local, está en alto riesgo ante el aumento de la intensidad de las tensiones.

El año 2012 finalizó bajo una frágil gobernabilidad. A los factores expuestos se suman otros. Los gobiernos municipales, nuevos actores de la joven democracia, son complacientes y refuerzan las lógicas de exclusión. El gobierno central continúa recurriendo a las estrategias de control político municipal por la vía de la compra de voluntades, la facilitación de proyectos y financiamientos, la intermediación política y financiera para aumentar sus apoyos a nivel territorial. Los alcaldes suelen ser actores importantes de estos conflictos, 
recibiendo presiones del gobierno central, del partido político al que pertenecen, de las empresas, de la oposición local y de su propio electorado. En ese juego de presiones, terminan cediendo al peso de los poderes económico y político tradicionales, que en diversas ocasiones combinan con los factores de poder emergentes, cuyos capitales proceden de las redes criminales y/o de los negocios con el Estado.

\section{CAMBIOS INSTITUCIONALES Y CONSTITUCIONALES}

En el marco de la promoción del desarrollo rural integral en Guatemala, a partir de la propuesta elaborada por las Universidades San Carlos de Guatemala y Rafael Landívar, orientada a replantear el proceso de discusión del desarrollo rural y buscar opciones para la viabilidad del proceso, presentada a los dos candidatos finalistas a la Presidencia de la República, encontró aceptación del candidato Otto Pérez Molina. El Presidente elegido incorporó el documento como fundamento para la creación de la Comisión Presidencial de Desarrollo Rural y para el Plan de Activación y Adecuación de la Política Nacional de Desarrollo Rural Integral (Acuerdo Gubernativo 196-2009). Posteriormente, tomó fuerza la discusión de la iniciativa 4084, Ley del Sistema de Desarrollo Rural Integral. Las organizaciones campesinas llevaron a cabo manifestaciones y otras acciones para presionar su pronta aprobación. Las cámaras empresariales reaccionaron en contra, generando un escenario de inviabilidad. Durante la primera semana de diciembre se tuvo la última oportunidad para su aprobación; incluso el Presidente de la República solicitó públicamente a su bancada apoyo al proyecto. Sin embargo, el resultado fue negativo. Las condiciones para 2013 son más complejas para retomar la discusión, ya que la comisión presidencial fue eliminada; así, no existe una instancia que promueva su retorno a la agenda política.

A inicio de 2011, la Universidad de San Carlos (USAC), la Asociación de Investigación y Estudios Sociales (ASIES) y la Universidad Rafael Landívar presentaron la propuesta de Reformas Constitucionales en materia de seguridad y justicia. Su finalidad es fortalecer las instituciones responsables de brindar seguridad y administrar justicia: Organismo Judicial, Ministerio Público, Instituto de la Defensa Pública Penal y Policía Nacional Civil. En 2012, cuando se creía que la propuesta había perdido impulso, el Presidente Pérez Molina presentó sorpresivamente una iniciativa para impulsar reformas constitucionales (reformas a 56 reformas). Después de una etapa de discusión con instancias de la sociedad civil, del ámbito empresarial, político y constitucional, se redujo el paquete de reformas (a 39 artículos), agrupándolas en siete ejes: sistema judicial, seguridad pública y ciudadana, democracia representativa, reconocimiento de la diversidad dentro de la unidad nacional, transparencia y rendición de cuentas, responsabilidad de funcionarios, asignación constitucional a las municipalidades, que se presentó al Congreso el 12 de agosto. La amplitud de temas, las dificultades para generar amplios consensos entre bancadas, así como la actitud del sector empresarial argumentando que buena parte de las reformas podían ser posibles mediante reformas a leyes ordinarias, crearon un ambiente negativo, que generó que el propio mandatario declinara en continuar impulsándolas. 
Otro proceso impulsado en 2012, sin mayores resultados, fue la discusión para promover nuevas reformas a la Ley Electoral y de Partidos Políticos. El proceso se inició en febrero con diversos espacios de diálogo entre la Comisión Específica de Asuntos Electorales del Congreso, Magistrados del Tribunal Supremo Electoral, sectores de la sociedad civil, partidos políticos y entidades internacionales especializadas. Las discusiones giraron en torno al fortalecimiento de los partidos políticos, la generación de mecanismos para garantizar mayor representatividad política de sectores subrepresentados, la necesidad de dotar de mayor capacidad sancionatoria al Tribunal Supremo Electoral y la readecuación de los plazos de los eventos electorales.

\section{PODER EJECUTIVO}

El gabinete que tomó posesión a partir del 15 de enero, refleja una composición donde domina la poca experiencia en la administración pública, la existencia de varios grupos que responden a intereses diversos (no solo del partido oficial) y la tendencia a generar agendas ministeriales con poca planificación, atención a la coyuntura y propensión a la generación de negocios.

De acuerdo con el Programa de Opinión Pública (POP), de la Universidad Rafael Landívar en el Informe de Evaluación del Primer Año de Gobierno, la calificación del trabajo realizado por los ministros fue calificada como positiva en aspectos generales. Esa percepción varía a partir de enero de 2013.

Tabla 1: Calificación del trabajo de los ministros

\begin{tabular}{|c|c|c|c|}
\hline Ministro y cartera & Muy malo & Malo & Bueno \\
\hline Efraín Medina, ministro de Agricultura (sustituido en enero de 2013) & & $X$ & \\
\hline Alejandro Sinibaldi, ministro de Comunicaciones & & & $X$ \\
\hline Carlos Batzín, ministro de Cultura y Deportes & & & $X$ \\
\hline Ulises Noé Anzueto Girón, ministro de Defensa Nacional & & & $X$ \\
\hline Sergio de la Torre, ministro de Economía & & & $X$ \\
\hline Cynthia del Águila, ministra de Educación & & & $X$ \\
\hline Erick Archila Dehesa, ministro de Energía y Minas & & $X$ & \\
\hline Pavel Centeno, ministro de Finanzas Públicas & & & $X$ \\
\hline Mauricio López Bonilla, ministro de Gobernación & & & $X$ \\
\hline Roxana Sobenes, ministra de Medio Ambiente y Recursos Naturales & & $X$ & \\
\hline Carlos Contreras, ministro de Trabajo y Prevención Social & & & $X$ \\
\hline $\begin{array}{l}\text { Harold Caballeros, ministro de Relaciones Exteriores (sustituido en } \\
\text { enero de 2013) }\end{array}$ & $X$ & & \\
\hline Jorge Villavicencio, ministro de Salud Pública & & $X$ & \\
\hline Luz Lainfiesta, ministra de Desarrollo Social & & & $X$ \\
\hline
\end{tabular}

Fuente: Elaboración propia del Programa de Opinión Pública (POP), Universidad Rafael Landívar, 2012. 
El Gabinete no tuvo cambios en el transcurso de 2012. A pesar de que desde los primeros meses se observó cierta separación entre los ministros cercanos a la figura presidencial, otros cercanos a la vicepresidenta, algunos con cercanía al sector empresarial, el mandatario asumió un papel activo al estar cerca de casi todos los temas de mayor visibilidad en la agenda pública.

\section{ORGANISMO LEGISLATIVO}

El Congreso de Guatemala es unicameral, integrado por 158 diputados elegidos para un período de cuatro años. La elección se lleva a cabo en el marco de las elecciones generales, que eligen al Presidente, Vicepresidente de la República y concejos de 334 municipios. A partir de la elección de 2003, ninguno de los partidos políticos ha dominado el organismo legislativo, debiendo establecer alianzas coyunturales con otras fuerzas políticas. Del total de escaños, 20 son ocupados por mujeres, que representan el 13 por ciento de los legisladores. Dos diputadas formaron parte de la Junta Directiva (de nueve miembros). Con un total de 11 diputadas, la Bancada Patriota es en la que participa el mayor número de mujeres.

El transfuguismo no es nuevo, pero se ha evidenciado con mayor fuerza en las últimas legislaturas. Solo en el primer año del Congreso más de un tercio del total de diputados cambiaron de partido o se declararon independientes (48, de 158).

Quince son los partidos políticos representados en el Congreso de la República, más un pequeño grupo de diputados que se han declarado independientes. Solamente tres partidos minoritarios (Encuentro por Guatemala, WINAQ y URNG) mantienen el mismo número de diputados que fueron elegidos en noviembre de 2011. La segunda fuerza con más votos (Unidad Nacional de la Esperanza, que gobernó en el período 2007-2011), pasó de 37 diputados (enero 2012) a 7 en enero 2013. El grupo parlamentario del partido LIDER ha sido el más beneficiado, pasando de 14 elegidos, a 36 (inicios de 2013). El partido oficial ha realizado alianzas tácticas con las bancadas de los partidos GANA, CREO, VIVA, y TODOS para aprobar iniciativas de interés del gobierno central. Con excepción de 4 bancadas minoritarias que se ubican como socialdemócratas o de tendencias asociadas a la Nueva Izquierda (UNE, WINAQ, EG y URNG), las restantes fuerzas políticas representan pensamiento conservador.

El papel de la oposición se resume a las acciones llevadas a cabo por la UNE y LIDER. Si bien al principio de la legislatura se estimaba que la primera de ellas encabezaría la oposición, debido a su desmembramiento acelerado capitalizado por LIDER, hizo que esta bancada sea la principal cuestionadora de las acciones del oficialismo. Las acciones de oposición se intensificaron en el segundo semestre de 2012, mediante acciones como romper el quórum, paralizar sesiones plenarias, alterar el orden del día, enfrentamientos directos entre diputados (agresiones físicas y verbales) y solicitud para llevar a cabo varias interpelaciones a ministros. De acuerdo con la Ley Orgánica del Congreso, las interpelaciones son un derecho de los representantes que impiden que se aborden otros asuntos en las sesiones plenarias. Esos ejercicios no tienen límite de duración, por lo que 
paralizan las labores legislativas; lo que sumado a las dificultades para lograr acuerdos interpartidarios, la carencia de agendas parlamentarias que superen la coyuntura y la dependencia que tiene el Legislativo respecto del Ejecutivo, crean un escenario de suma dificultad.

Tabla 2: Conformación del Congreso de la República, enero de 2012 vrs. enero de 2013

\begin{tabular}{lcc}
\hline & Diputados elegidos & Diputados actuales \\
\hline Patriota & 57 & 60 \\
Partido Libertad Democrática (LIDER) & 14 & 36 \\
Unidad Nacional de la Esperanza (UNE) & 36 & 7 \\
Unión del Cambio Nacional (UCN) & 14 & 3 \\
Compromiso, Renovación y Orden (CREO) & 12 & 8 \\
Gran Alianza Nacional (GANA) & 12 & 8 \\
PAN & 2 & 1 \\
Encuentro por Guatemala (EG) & 3 & 3 \\
WINAQ-AN & 1 & 1 \\
Frente Republicano Guatemalteco (FRG, hoy PRI) & 1 & 1 \\
Unidad Revolucionaria Nacional Guatemalteca (URNG) & 1 & 1 \\
Unionista & 1 & 3 \\
Victoria & 1 & 2 \\
VIVA & 3 & 17 \\
Todos & 0 & 6 \\
Independientes & 0 & \\
\hline
\end{tabular}

Fuente: Elaboración propia a partir de datos de la Dirección Legislativa del Congreso de la República.

El Congreso cuenta con 46 comisiones de trabajo. En la legislatura 2012-2013, cuatro fueron ocupadas por mujeres y cinco por indígenas.

\section{CRECIENTE PÉRDIDA DE AUTONOMÍA}

Se ha restado funcionalidad al Legislativo en el marco de la imposición de prioridades desde el Ejecutivo, que a su vez tiene dos referentes: i) la agenda presidencial y de su círculo de influencia, ii) los temas de interés de la vicepresidencia, quien además es secretaria general del Partido Patriota. Esa supeditación quedó en evidencia en los dos momentos de mayor actividad legislativa: la primera, en sus inicios, que permitió la aprobación de la ley que contiene reformas fiscales, la creación del Ministerio de Desarrollo Social y el Protocolo de Roma y, la segunda, que permitió la rápida aprobación del presupuesto general de ingresos y egresos 2013, la nueva Junta Directiva que tomó posesión el 14 de enero de 2013, dos préstamos para las entidades consentidas de la Presidencia (Ministerio de la Defensa y Ministerio de Comunicaciones), entre otros temas de interés. 
En el caso del Organismo Judicial (OJ), los señalamientos de poca efectividad, dificultades para la coordinación con otros entes públicos y las denuncias en contra de operadores de justicia dominó la agenda en 2012. Se crearon juzgados que por primera ocasión conocerán de casos de femicidio y trata de personas. Se continúan los esfuerzos para aumentar la cobertura jurisdiccional en todo el territorio. Los delitos contra la vida, los casos asociados a la criminalidad (narcotráfico, pandillas, extorsiones, redes de trata), así como las denuncias por acciones de corrupción, llenan de expedientes a los operadores tanto del organismo como del Ministerio Público. El OJ constantemente sugiere el aumento de su presupuesto como condición básica para ser más eficiente. En 2012, en el marco de la propuesta presidencial de reformas constitucionales, el OJ manifestó su disposición en el sentido de reformar artículos específicos que procuren mejorar su accionar judicial.

\section{SUJECIÓN Y PRÁCTICAS CLIENTELARES}

La Constitución reconoce dos niveles de gobierno, el nacional y el local (municipal). El país cuenta con 334 municipios. A pesar de la existencia de la autonomía municipal, como forma de organización preceptuada por el Código Municipal, las autoridades locales han reforzado su dependencia del gobierno central. La debilidad en los mecanismos de diálogo político entre ambos niveles contribuye a incrementar los programas clientelares, como estrategias para la compra de voluntades, el debilitamiento de cualquier movimiento de oposición, el uso indiscriminado de la relación entre fondos públicos y contratos de obra física como mecanismo de control político, que además ha producido la irrupción de un sector económico fincado a nivel local, cuya riqueza es posible a costa de los recursos públicos. Ese sector emergente también representa un actor político en aumento, en la medida en que no solo apelan a controlar negocios, sino también las alcaldías municipales y las delegaciones de las dependencias del gobierno central que tienen mayor presencia territorial y recursos.

\section{VII. ¿HACIA DÓNDE SE ORIENTA LA DINÁMICA POLÍTICA? A MANERA DE SÍNTESIS Y CONCLUSIONES}

Guatemala se debate entre tres factores clave: 1) recambio en los factores de poder económico. De la oligarquía tradicional que hegemonizó la estructura económica productiva, se ha pasado a la coexistencia entre el capital tradicional, el emergente lícito, el emergente ilícito y quienes se han enriquecido por medio de expoliar los recursos públicos. Estos tres últimos se fortalecen a costa del recorte de los márgenes de maniobra del tradicional, principales impulsores de los proyectos de explotación de recursos naturales. Las dificultades a las que se enfrenta ese tipo de actividades productivas se entiende, en parte, por el peso, capacidad de articulación territorial y mayor dinámica de las otras expresiones del capital. Sin embargo, no se trata de la modificación del modelo de desarrollo o la emergencia de nuevos actores políticos; 2) el agotamiento de los partidos políticos, como principales canales de intermediación política, ha generado 
un escenario de enfrentamiento directo entre los actores económicos y quienes se oponen a los proyectos estratégicos. Las instituciones están sobrepasadas en sus capacidades de articulación e impulsoras de procesos de diálogo. Ese factor, sumado a la insuficiente capacidad del gobierno para traducir las demandas del complejo social; 3) la única posibilidad de interrelación entre los capitales en pugna es el objetivo de repartir los recursos del Estado para reproducir el sistema. Ese vínculo termina siendo el "factor de oportunidad" para impulsar la viabilidad de los proyectos. Este marco coloca en cuestión los dos criterios planteados por Karl Deutsch ${ }^{1}$ para analizar la estructura de los Estados: alcance (variedad de actividades humanas que trata de controlar) y dominio (territorio y población controlados). La capacidad de control del Estado se ha debilitado, espacio que es aprovechado por poderes fácticos para poner más en riesgo la precaria gobernabilidad.

Una de las expresiones de esa lógica de sustitución de capacidades del Estado radica en la mutación que ocurre en el sistema de partidos. Más que debilitamiento del sistema, lo que se observa es la pérdida acelerada de la relación pesos-contrapesos en el organismo legislativo y el incremento de las dependencias de los partidos allí representados, de sectores privados que operan y se enriquecen con los fondos públicos. Contrario a la concepción del estado de derecho en sociedades exitosas, donde los partidos contribuyen a su estabilidad, intermediación política, predisposición al debate y empoderamiento de la sociedad, los partidos guatemaltecos son factores de desconfianza, desarrollan sistemas paralelos que compiten con la autoridad del Estado, permiten el debilitamiento de las finanzas públicas y se sienten superiores a la ley. ${ }^{2}$ La deserción (transfuguismo) es solo una de las tácticas utilizadas por los legisladores para trazar rutas que permitan mantener a flote la prevalencia del clientelismo y corporativismo acelerado.

Desde 2009 se ha escuchado con frecuencia que Guatemala está catalogado como un Estado fallido, o a punto de serlo. De las diez funciones fundamentales para evaluar esa condición, sugeridas por Ashraf Ghani, ${ }^{3}$ el peso de la discusión se centra en dos: estado de derecho y monopolio del uso de la fuerza. En el primer caso, a pesar que el desempeño de los indicadores en materia de estado de derecho, deslegitimización del Estado y violación de derechos humanos es menor al promedio latinoamericano, ha habido mejoras considerables desde 2010 al momento actual. En el segundo caso, el país muestra mejora a lo largo del tiempo especialmente en los indicadores de estabilidad política y ausencia de violencia, intensidad del conflicto, incidencia de golpes de Estado, refugiados, y aparatos de seguridad. La mejora en los indicadores de incidencia de la criminalidad, reflejados de 2009 a 2012, ayudan en esa materia. Las redes criminales no controlan la totalidad del territorio; las luchas entre carteles y grupos de narcotraficantes están focalizadas en áreas fronterizas. La captura y extradición de narcos locales solicitados por la justicia de Estados Unidos es otro dato positivo, así como la continuidad de los programas de decomiso de drogas.

Karl Deutsch. Política y gobierno (México, Fondo de Cultura Económica, 1976).

Asociación de Investigación y Estudios Sociales (ASIES). Guatemala no es un Estado fallido. Guatemala, 2010.

Ashraf Ghani and Clare Lockhart. Fixing failed states: a framework for rebuilding a fractured world. Oxford University Press. 2008. 
Los escenarios de la conflictividad han aumentado en número y diversidad de fundamentos. La presencia de movilizaciones contra proyectos extractivos e hidroeléctricos, impulsadas especialmente por comunidades indígenas, se enmarca en un proceso de conflictividad social donde el actor indígena quiere ser protagonista como actor político que no ha saltado a la palestra a consecuencia de un sistema político excluyente. El Estado guatemalteco no ha logrado construir las condiciones mínimas para que el liderazgo político pueda discernir y responder a los nuevos patrones de demanda, que combina demandas sociales, mayores espacios políticos con cuestionamientos crecientes del modelo de desarrollo basado en commodities, su carácter extractivo y los cambios que genera en las dinámicas del territorio.

La legitimidad de esas demandas de organizaciones comunitarias ha quedado en segundo término. En principio, la razón inicial de las oposiciones es elevar los grados de tensión hasta donde sea posible, para aprovechar las debilidades de las instituciones de gobierno y generar un estado permanente de ingobernabilidad. Las acciones de rechazo y descalificación, si no están acompañadas de proyecto político, solo generan que sus planteamientos adquieran mayor resonancia.

Las necesidades y oportunidades de desarrollo se han invisibilizado cuando hacen su aparecimiento intereses de terceros. Es importante crear mecanismos de diálogo directos, para que las demandas y realidades legítimas de las comunidades no se presten a malos manejos. Los discursos de fácil consumo, acompañados de recursos externos para azuzar, han aumentado en los últimos 3 años, creando mayores incentivos para el aumento de las tensiones, para unificar y fortalecer las resistencias a los proyectos relacionados con recursos naturales. Resulta clave desactivar los focos de ingobernabilidad.

En la medida que la presencia del Estado y sus instituciones a nivel territorial sea escasa, que predominen las condiciones de pobreza y extrema pobreza, que el flujo de recursos financieros sea sostenible para promover movilizaciones y planteamientos de fácil consumo y se mantenga la percepción adversa (nacional e internacional) sobre el país, las condiciones para las inversiones privadas en recursos naturales seguirán siendo difíciles.

\section{REFERENCIAS}

Ashraf Ghani and Clare Lockhart. 2008. Fixing failed states: a framework for rebuilding a fractured world. Oxford: Oxford University Press.

Asociación de Investigación y Estudios Sociales (ASIES). 2012. Monografía de partidos políticos de Guatemala, 2012. Iniciativa Think Tank. Fundación Konrad Adenauer. Centro de Impresiones Gráficas -CIMGRA. Asociación de Investigación y Estudios Sociales (ASIES). 2010. Guatemala no es un Estado fallido. Guatemala. Congreso de la República. 2012. Decreto No 10-2012. Ley de Actualización Tributaria.

Congreso de la República. 2012. Decreto N ${ }^{o} 30-2012$. Ley de Presupuesto General de Ingresos y Egresos del Estado. Dinorah Azpuru. 2012. Cultura política de la democracia en Guatemala y en las Américas, 2012. Hacia la igualdad de oportunidades. Vanderbilt University. Asociación de Investigación y Estudios Sociales (ASIES).

Dirección de Incidencia Pública. 2011. Informe Final, proyecto de investigación Poder Local, Incidencia Política y Gobernabilidad en temas de Justicia ambiental, defensa del territorio y derechos de los pueblos indígenas en Guatemala. Guatemala: Universidad Rafael Landívar.

Karl Deutsch. 1976. Política y gobierno. México: Fondo de Cultura Económica. 
Mendoza, Carlos. 2012. Informe Homicidios en Guatemala. Central American Business Intelligence (CABI). Observatorio de Ética y Justicia. 2012. Responsabilidad disciplinaria de Jueces y Magistrados de Guatemala. Primer Informe. Guatemala: Universidad Rafael Landívar.

Observatorio de Ética y Justicia. 2012. Responsabilidad disciplinaria de Jueces y Magistrados de Guatemala. Segundo Informe. Guatemala: Universidad Rafael Landívar.

Partido Patriota. 2011. Agenda del Cambio. Plan de Gobierno 2012-2016.

Ministerio de Gobernación. 2012. Pacto por la Seguridad, Justicia y Paz. Gobierno de la República de Guatemala.

Programa de Naciones Unidas para el Desarrollo (PNUD). Informe de Desarrollo Humano 2012 (en línea) http//:www.hdr.undp.org.

Programa de Opinión Pública (POP). 2012. Informe del Primer Año de Gobierno de Otto Pérez Molina. Guatemala: Universidad Rafael Landívar.

Rosal, Renzo. 2012. Presente y futuro de la minería en Guatemala, un debate en desarrollo. Revista P@x. Universidad de Coimbra.

Secretaría de Planificación y Programación de la Presidencia. 2012. Pacto Hambre Cero: Retos para Guatemala. Gobierno de la República de Guatemala.

Universidad San Carlos de Guatemala, Universidad Rafael Landívar. 2011. Propuesta para abordar el Desarrollo Rural Integral de Guatemala.

Secretaría de Planificación y Programación de la Presidencia (Segeplan). 2011. Informe del Primer Año de Gobierno. Informe del Presidente al Congreso de la República. Guatemala.

Renzo Lautaro Rosal. Politólogo con especialización en Sociología Política. Tiene una Maestría en Relaciones Internacionales (FLACSO/Universidad Rafael Landívar). Actualmente es Director de Incidencia Pública de la Universidad Rafael Landívar. Presidente del Consejo Editorial del periódico digital Plaza Pública. Columnista en el periódico Prensa Libre. Investigador de procesos electorales y políticas públicas. Analista político. Consultor internacional en descentralización y fortalecimiento de gobiernos locales. Funcionario público en varias administraciones de gobierno, habiendo sido Subsecretario Ejecutivo de la Presidencia, responsable del proceso de descentralización. Docente universitario.

E-mail: renzolautaro.rosal@gmail.com 